\title{
EFFECTS OF SMALL-SIDED GAMES AND CONVENTIONAL AEROBIC INTERVAL TRAINING ON VARIOUS PHYSIOLOGICAL CHARACTERISTICS AND DEFENSIVE AND OFFENSIVE SKILLS USED IN SOCCER
}

\author{
İlker Özcan, Niyazi Eniseler, and Çağatay Şahan \\ Faculty of Sports Sciences, Celal Bayar University, Manisa, Turkey
}

Original scientific paper

https://doi.org/10.26582/k.50.1.12

UDC: $796.332: 796.012 .2$

\begin{abstract}
:
The aim of this study was to investigate the effects of small-sided game training (SSGT) versus conventional aerobic interval training (CAIT) on soccer-specific endurance performance, lactate threshold levels $\left(\mathrm{mmol} \cdot \mathrm{L}^{-1}\right)$, short-passing ability and defensive and offensive skills of a soccer match. Before and after a 6-week training intervention period, eighteen amateur soccer players (age $21.8 \pm 4.8$ years) were tested. The tests included the anaerobic threshold (AnT) test, the Yo-Yo Intermittent Recovery Test Level 1 (Yo-Yo IR1), and the Loughborough Soccer Passing Test (LSPT). A manual notational match analysis system was utilized to evaluate the defensive and offensive skills of players during the matches. Both the SSGT and CAIT were performed two days a week and consisted of five sets of 6-minute periods of work at the individualized exercise intensity corresponding to the individual anaerobic threshold, with 3-minute recovery periods between sets. Mann-Whitney U and Wilcoxon signed rank tests were used to examine the between- and within-group differences, respectively. Statistical analyses revealed that the SSGT group players exhibited significantly better in terms of the LSPT scores $(\mathrm{p}<.01)$ and the number of defensive and offensive skills $(p<.05)$. However, no other significant differences in the other variables were observed $(p>.05)$. The results of the study suggested that SSGT improved short-passing ability, various soccer skills and physiological parameters, while CAIT only improved physiological parameters. SSGT improves soccer-specific endurance and technical ability of players at the same time, meaning it is a time efficient way of training.
\end{abstract}

Key words: small-sided game, anaerobic threshold training, soccer skills, short-passing ability

\section{Introduction}

Football performance is dependent on a multitude of factors. Technical skills (Ali, 2011), playing tactics (Reilly, 1996; Reilly \& White, 2005) and endurance capacity (Bangsbo, Iaia, \& Krustrup, 2008; Dellal, et al., 2008) are known to have major influences on match performance. For this reason, aerobic endurance training is an important component of physical conditioning in soccer (Dellal, et al., 2008) besides technical and tactical skills that need to be developed to improve soccer performance. Although Helgerud, Engen, Wisløff, and Hoff (2001) have demonstrated the effectiveness of running interval training, small-sided games (SSGs) are often used as an alternative to generic interval training to provide an aerobic training stimulus for soccer players (Little \& Williams, 2006; Rampinini, et al., 2007). One of the main differences between these two training methods is that the presence of the ball during small-sided games allows the concomitant improvement of tech- nical and tactical skills and enhances motivation of players (Flanagan \& Merrick, 2002). Additionally, if running drills without the ball are used to improve endurance capacities, players will need extra practice time to improve their technical skills (Hoff, Wisløff, Engen, Kemi, \& Helgerud, 2002).

Elite soccer clubs often have busy schedules, due to which coaches struggle to integrate different training parameters. Coaches have a limited amount of time to work with their athletes during the preseason and, especially, in-season. An experienced coach preparing for a competition can optimize limited work time by integrating technical, tactical and physiological training contents into the planned cycles. Such a holistic approach favors the use of exercises with the ball as much as possible (Reilly, 1996). Physical conditioning using soccer games would be an extremely effective use of training time and physical load.

Many authors investigated the effects of smallsided game training (SSGT) and conventional 
aerobic interval training (CAIT) on soccer-specific physical performance (Ferrari Bravo, et al., 2008; Helgerud, et al., 2001; Hill-Haas, Coutts, Rowsell, \& Dawson, 2009; Impellizzeri, et al., 2006; Radziminski, Rompa, Barnat, Dargiewicz, \& Jastrzebski, 2013). These studies have shown that each type of training method has the potential to improve players' aerobic endurance. Although every critic and researchers had stated that SSGT develops technical skills and playing tactics (Allison \& Thorpe, 1997; Radziminski, et al., 2013; Reilly \& White, 2005), no one has yet managed to provide accurate and valid data to measure technical and tactical improvement after the small-sided game training. Therefore, the aim of this study was to investigate the effects of SSGT versus CAIT on the development of soccer-specific defensive and offensive game skills and short-passing abilities, then on soccer-specific endurance performance and lactate threshold level measures.

Various studies suggest that SSGs, being more specific to match demands, induce technical, physical and tactical implications for each player offensively and defensively (Bekris, et al., 2012; Da Silva, et al., 2011; Dellal, et al., 2012; Fanchini, et al., 2011; Martone, et al., 2017). Thus, the hypothesis was that SSGT would have significantly greater effects than CAIT on soccer-specific endurance performance, defensive and offensive game skills and short-passing abilities.

\section{Methods}

\section{Procedures}

The subjects were randomly allocated to either the SSGT group (SSGTG) or CAIT group (CAITG). The experimental design is presented in Table 1. Before and after the intervention period, anthropometrical assessments, the Yo-Yo Intermittent Recovery Test Level 1 (Yo-Yo IR1) and anaerobic threshold (AnT) tests were used to measure players' physical capacities, while the Loughborough Soccer Passing Test (LSPT) and notational analysis system was used to measure short-passing abilities and offensive and defensive skills during soccer matches, respectively. All the tests were performed in the morning. During the study, all the players were instructed to maintain their regular daily food and water intakes, and no dietary interventions were conducted. The players were asked to consume their usual meal at least three hours before the scheduled testing time.

\section{Participants}

Eighteen male soccer players participated in this study. The characteristics of the participants are presented in Table 2. The male soccer players were from an amateur team that competed at the regional level. The players practiced approxi- mately six hours per week, which was equivalent to four days of training and one competition game per week. The sample of participants included all playing positions, with the exception of the goalkeeper. The protocol of the study was approved by the Ethical Committee of the Celal Bayar University according to the revised Declaration of Helsinki before the commencement of the assessments. Written informed consent forms were received from all players after a brief, but detailed explanation of the aims, benefits and risks of the study. All players were informed that they could withdraw from the study at any time without any penalty.

\section{Anthropometrical assessments}

Anthropometrical measurements included body height, body mass and the sum of four skinfold measures (the triceps, subscapular, abdominal, and suprailiac sites) taken by Harpenden calipers, which was used to determine body fat percentages (Yuhasz, 1962).

\section{Yo-Yo Intermittent Recovery Test Level 1}

The Yo-Yo IR1 test was performed as described by Krustrup et al. (2003). The audio cues for the Yo-Yo IR1 were recorded on a CD (Teknosport ${ }^{\mathrm{TM}}$ ). The test was terminated when the participant stopped voluntarily or failed twice to reach the front line in time with the audio cue. The total distance covered during the Yo-Yo IR1 (including the last incomplete shuttle) was taken as the testing result.

\section{Anaerobic Threshold Test}

An incremental treadmill running test was applied to identify the running velocity (vOBLA) and heart rate (OBLAHR) that corresponded to the fixed blood lactate concentration of $4 \mathrm{mmol} \cdot \mathrm{L}^{-1}$. Specifically, in this test, the initial running speed was eight $\mathrm{km} \cdot \mathrm{h}^{-1}$, and the treadmill grade was set at $1 \%$. Speed was increased to $10 \mathrm{~km} \cdot \mathrm{h}^{-1}$ in the second stage and later it was increased by $1 \mathrm{~km} \cdot \mathrm{h}^{-1}$ every three minutes until volitional exhaustion (Guner, Kunduracioglu, \& Ulkar, 2006). Between running stages, 30 -second pauses were allowed for the collection of 25- to 40-microlitre capillary blood samples from the fingertip by heparinized pipettes for lactate analyses. The blood samples were analyzed for lactate concentration with an electro-enzymatic method (YSI 1500 Sport Lactate Analyzer, Yellow Springs Instrument Co., Yellow Springs, $\mathrm{OH}$ ). The subjects' heart rates were measured by Polar RS400 telemetric heart rate monitors (Polar Electro Oy, Kempele, Finland). The lactate concentrations and heart rate values were recorded for each running speed. Running velocities (vOBLA) and heart rates (OBLAHR) that corresponded to the blood lactate concentration of $4 \mathrm{mmol} \cdot \mathrm{L}^{-1}$ were calculated using a spline function (Guner, et al., 2006). 


\section{Loughborough Soccer Passing Test (LSPT)}

To obtain an objective measure of short-passing ability, the first version of the LSPT was used. This version has been shown to be both reliable and valid (Ali, et al., 2007). The detailed protocol and a schematic representation of the test have been presented in Ali et al. (2007). The participants performed the test twice, and the mean was taken as their performance score. The final LPST performance score has been named the LPST time (in seconds) (Ali, et al., 2007).

\section{Offensive and defensive skills}

The players participated in two 11-a-side friendly matches that were played with no substitutions on the same surface area $(105 \times 70 \mathrm{~m})$. The extra-times in each half were excluded from the analysis; thus, only $90 \mathrm{~min}$ of the activities were recorded. Video recordings were analyzed for defensive and offensive skills performed during each match. For the analysis, a manual notational analysis system was developed based on the OPTA Client System which has been proven reliable and valid (Liu, Hopkins, Gomez, \& Molinuevo, 2013). The actions coded by the operator were analyzed in two skill groups: offensive (OS) and defensive (DS). Operational definitions of the variables are explained below. Offensive skills were: pass (the ball intentionally played from a player to his teammate), dribble (an attempt by a player to beat an opponent by driving the ball), ball control (directing the ball into space away from the body, stopping or settling the ball at one's feet). The defensive skills were: tackle (act of [re]gaining possession from the opposing player, winning the ball), interception (stepping into a pass line) and aerial duel (competing for the ball in the air) (Liu, Gomez, Lago-Peñas, \& Sampaio, 2015; Liu, et al., 2013; OPTA, 2012). The operator was required to learn the definitions of variables thor- oughly in order to get full awareness of coding for reliability (Bradley, O'Donoghue, Wooster, \& Tordoff, 2007; O’Donoghue, 2007).

\section{Training intervention program}

The weekly schedule of the training intervention is shown in Table 1. The SSGT or CAIT was added to the regular soccer training sessions (60-90 min). The 6-week training intervention spanned four weeks of the pre-season and two weeks of the competitive season. During two weeks prior to the training intervention, the players performed general conditioning to prevent possible injuries. The CAIT and SSGT intervention programs were always performed at the beginning of the session, following a standardized warm-up. No other endurance training program was completed during the study period.

The SSGs were organized into 6-a-side and 5 -a-side teams (no goalkeepers) on a playing field within the areas of $36 \mathrm{~m} \times 48 \mathrm{~m}$ and $30 \mathrm{~m} \times 42 \mathrm{~m}$ during the first and second three weeks, respectively (Rampinini, et al., 2007). The extra players needed for 6-a-side and 5-a-side teams were chosen from players who were not participating in the study.

The number of players and the size of game area for the SSGT were chosen specifically to allow the players to reach the targeted intensity (HR) which was under constant surveillance (Ian, 2004). During the intervention, the CAITG ran intervals of 6 minutes at speed that kept the players at their lactate threshold level. The interval workout was executed on the grass around the soccer field without a ball.

During the interventions, both the SSGTG and CAITG trained at individualized exercise intensities. The heart rates ( \pm 5 beats/min) corresponding to the lactate threshold level of $4 \mathrm{mmol} \cdot \mathrm{L}^{-1}$ (OBLAHR) were used as the individualized exercise intensity goals during the sessions (Eniseler, 2005). The individualized training intensities for each session

Table 1. Pre-test, post-test and 6-week periodized SSGT and CAIT interventions

\begin{tabular}{llllllll}
\hline Week & Monday & Tuesday & Wednesday & Thursday & Friday & Saturday & Sunday \\
\hline Pre-test & & & LSPT+Yo-Yo IR1 & Rest & Test match & Rest & AnT test \\
Week1 & Day off & Str+Inter & Agility +Tact & Inter+Tact & Tech & Day off & F match \\
Week2 & Day off & Str+Inter & Agility+Tact & Inter+Tact & Speed+Tech & Day off & F match \\
Week3 & Day off & Str+Inter & Agility+Tact & Inter+Tact & Speed+Tech & Day off & F match \\
Week4 & Day off & Str+Inter & Agility+Tact & Inter+Tact & Speed+Tech & Day off & F match \\
Week5 & Day off & Str+Inter & Agility+Tact & Inter+Tact & Speed+Tech & Day off & O\&F match \\
Week6 & Day off & Str+Inter & Agility+Tact & Inter+Tact & Speed+Tech & Day off & O\&F match \\
Post-test & Day off & LSPT+ & Rest & Test match & Rest & AnT test & \\
& & Yo-Yo IR1 & & & & & \\
\hline
\end{tabular}

Note. LSPT=Loughborough Soccer Passing Test; Yo-Yo IR1=the Yo-Yo Intermittent Recovery Test Level 1; AnT test=anaerobic threshold test; Str=strength (low-level prevention sessions); Inter=CAIT (conventional aerobic interval training) or SSGT (smallsided game training); Tact=tactical session (low intensity); Tech=technical session (goal-scoring); F match=friendly match; O\&F match $=$ official and friendly match. 
were controlled and optimized with the real-time heart rate feedback (the coach warned the players if immediate HR adjustment was needed) using a short-range radio telemetry during the SSGT (Activio Fitness System, Nordenflychtsvagen 66, SE-112 51 Stockholm, Sweden) and CAIT (Polar RS400, Finland).

Both the SSGT and CAIT were performed two days a week and consisted of five 6-minute sets of work at the individualized exercise intensity, with 3 -minute recovery periods between sets. The participants were well familiarized with all of the CAIT and SSGT formats.

The $5^{\text {th }}$ and the $6^{\text {th }}$ week of the program included official games (Table 1). For the players who did not participate in the official games, two friendly games were conducted in order to equalize the number of games per player. Other than the intervention programs, the volume of exercise and the training contents of practices were the same for all players.

\section{Offensive skills in SSGT}

The notational analysis system (explained in the section Offensive and defensive skills in the matches) was also used to evaluate offensive skills executed in the SSGTs in order to emphasize importance of work with the ball.

\section{Statistical analysis}

The statistical package SPSS (Statistical Package for Social Science, 16.0) was used for the analysis. Data are expressed as mean \pm standard deviation. The Kolmogorov-Smirnov test was first applied to confirm distribution normality of the data and Levene's test was applied to assess the homogeneity of variance. Because of the limited number of participants and the lack of homogeneity in groups, Wilcoxon Signed Ranks and Mann-Whitney U test were used to examine the differences between the training effects and groups. Significance level was defined as $\mathrm{p} \leq .05$. Effect size (ES) for independent samples Wilcoxon Signed Ranks and MannWhitney U tests were calculated using Cohen's d statistic (Cohen, 1988). Values of 0.1, 0.3 and $>0.5$ were considered small, medium and large, respectively (Coolican, 2009).

\section{Results}

There were no significant differences in the baseline anthropometric parameters between the groups ( $>.05$, Table 2). After 6 weeks of intervention, body mass values and body fat percentages did not change significantly in CAITG ( $p>.05$, Table 3), while there were small to large effects on body mass and percentage of body fat in SSGTG

Table 2. Descriptive characteristics of the participants

\begin{tabular}{|c|c|c|c|c|c|}
\hline & $\begin{array}{l}\text { Body mass } \\
(\mathrm{kg})\end{array}$ & $\begin{array}{l}\text { Body height } \\
\text { (m) }\end{array}$ & $\begin{array}{c}\text { Age } \\
\text { (years) }\end{array}$ & $\begin{array}{l}\text { Body fat } \\
\text { (\%) }\end{array}$ & $\begin{array}{c}\text { Training experience } \\
\text { (years) }\end{array}$ \\
\hline CAITG $(n=9)$ & $68.36 \pm 8.93$ & $1.75 \pm 10.06$ & $18.54 \pm 1.54$ & $11.29 \pm 0.89$ & $3.77 \pm 1.90$ \\
\hline SSGTG $(n=9)$ & $62.70 \pm 6.14$ & $1.71 \pm 6.05$ & $18.43 \pm 1.47$ & $10.73 \pm 1.18$ & $4.22 \pm 2.27$ \\
\hline
\end{tabular}

Note. CAITG=conventional aerobic interval training group; SSGTG=small-sided game training group.

Table 3. Effects of SSGT and CAIT. ${ }^{*} p<.05 ; *^{*} p<.01$, significantly different from the pre-intervention value

\begin{tabular}{|c|c|c|c|c|c|c|c|c|c|c|}
\hline \multirow[t]{2}{*}{ Variables } & \multicolumn{2}{|c|}{ CAITG $(n=9)$} & \multicolumn{8}{|c|}{ SSGTG $(n=9)$} \\
\hline & Pre & Post & (\%) & $P$ & ES & Pre & Post & (\%) & $P$ & ES \\
\hline $\begin{array}{l}\text { Body mass } \\
(\mathrm{kg})\end{array}$ & $68.36 \pm 8.93$ & $67.61 \pm 9.69$ & -1.21 & 0.176 & 0.31 & $62.70 \pm 6.14$ & $61.81 \pm 5.66^{*}$ & -1.36 & 0.021 & 0.54 \\
\hline Body fat (\%) & $11.29 \pm 0.89$ & $10.88 \pm 1.12$ & -3.65 & 0.123 & 0.36 & $10.73 \pm 1.18$ & $10.00 \pm 0.98^{*}$ & -6.54 & 0.011 & 0.14 \\
\hline $\begin{array}{l}\text { vOBLA } \\
\left(\mathrm{km}^{2} \mathrm{hr}^{-1}\right)\end{array}$ & $10.6 \pm 1.5$ & $11.8 \pm 1.5^{* *}$ & +12.22 & 0.008 & 0.62 & $10.8 \pm 0.8$ & $12.3 \pm 0.6^{* *}$ & +14.84 & 0.008 & 0.62 \\
\hline $\begin{array}{l}\text { Yo-Yo IR1 } \\
\text { (meter) }\end{array}$ & $1057.7 \pm 359.5$ & $1826.6 \pm 432.6^{*}$ & +89.09 & 0.012 & 0.59 & $1235.5 \pm 367.9$ & $1946.6 \pm 550.9^{* *}$ & +63.13 & 0.008 & 0.62 \\
\hline $\begin{array}{l}\text { LSPT time } \\
\text { (second) }\end{array}$ & $54.4 \pm 8.1$ & $57.5 \pm 13.9$ & +5.33 & 0.594 & 0.13 & $59.21 \pm 8.65$ & $46.71 \pm 3.62^{* *}$ & +19.56 & 0.008 & 0.62 \\
\hline $\begin{array}{l}\text { The number } \\
\text { of defensive } \\
\text { skills }\end{array}$ & $17 \pm 9.8$ & $12.1 \pm 4.9$ & -15.53 & 0.109 & 0.37 & $14.4 \pm 6.6$ & $20 \pm 6.6^{*}$ & +64.70 & 0.023 & 0.53 \\
\hline $\begin{array}{l}\text { The number } \\
\text { of offensive } \\
\text { skills }\end{array}$ & $59.2 \pm 26.5$ & $70.8 \pm 23.2$ & +32.67 & 0.123 & 0.36 & $58.2 \pm 19.2$ & $78.2 \pm 30.9^{*}$ & +33.02 & 0.012 & 0.59 \\
\hline
\end{tabular}

Note. CAITG=conventional aerobic interval training group; SSGTG=small-sided game training group; vOBLA=running velocities that corresponded to blood lactate concentrations of $4 \mathrm{mmol} \cdot \mathrm{L}^{-1}$; Yo-Yo IR1= the Yo-Yo Intermittent Recovery Test Level 1; LSPT=Loughborough Soccer Passing Test; ES=effect size. 
(Table 3). Both the SSGT and CAIT induced significant improvements in vOBLA ( $p<.01$, large effects, Table 3). The distance covered during the Yo-Yo IR1 test increased significantly in SSGTG $(p<.01$, large effect) and CAITG ( $p<.05$, large effect, Table 3). The LSPT performance score in SSGTG showed significant improvements $(\mathrm{p}<.01$, large effect, Table 3), while no improvement was observed in CAITG (Table 3). Significant improvements in the number of defensive and offensive skills executed during soccer matches in SSGTG were also observed $(\mathrm{p}<.05$, large effect, Table 3$)$. The number of defensive skills during soccer matches in CAITG decreased significantly $(\mathrm{p}<.05$, medium effect), while there was no significant increase in the number of offensive skills ( $p>.05$, Table 3 ).

\section{Discussion and conclusions}

The present study used both SSGs and CAIT as soccer training methods to improve aerobic endurance of participants. The effects of SSGT and CAIT on aerobic performance have already been compared in elite soccer players (Ferrari Bravo, et al., 2008; Hill-Haas, et al., 2009; Impellizzeri, et al., 2006; McMillan, Helgerud, Macdonald, \& Hoff, 2005; Radziminski, et al., 2013; Reilly \& White, 2005), but few studies have attempted to determine whether SSGT can improve soccer game skills and technical ability (Radziminski, et al., 2013; Reilly $\&$ White, 2005). Thus, the aim of this study was to determine the effects of 6-week SSGT and CAIT interventions on soccer-specific endurance measures, defensive and offensive game skills and shortpassing abilities among amateur soccer players. The main findings revealed that both the SSGT and CAIT interventions significantly improved the players' physical fitness as assessed by vOBLA and Yo-Yo IR1 performance measures. As expected, the SSGTG exhibited improvements in defensive and offensive game skills and short-passing abilities, whereas the CAITG did not.

Endurance capacities of soccer players are generally trained using running drills that do not involve the ball. A major reason for this is that soccer games are believed not to provide sufficient exercise intensity, i.e. training stimulus, to effectively improve the physiological mechanisms important for soccer endurance (Helgerud, et al., 2001; Wisløff, Helgerud, \& Hoff, 1998). However, researchers (Allison \& Thorpe, 1997; Hoff, et al., 2002; Sassi, Reilly, \& Impellizzeri, 2005) have observed that exercise intensities that have been deemed appropriate for soccer endurance training occur during various SSGs.

Furthermore, various studies have reported that both high-intensity SSGT and generic interval training (i.e., $4 \times 4$-minute drills at $90-95 \%$ of the HRmax with 3 -minute active recovery periods, twice per week) elicited similar changes in endur- ance performance in soccer players, i.e., lactate thresholds, soccer match physical performance and Yo-Yo IR1 performances; (Chamari, et al., 2005; Ferrari Bravo, et al., 2008; Helgerud, et al., 2001; Hill-Haas, et al., 2009; Impellizzeri, et al., 2006; McMillan, et al., 2005; Reilly \& White, 2005). All of the mentioned studies, including our own, have demonstrated that the SSGT and CAIT methods both have potential to improve players' endurance capacities.

Although the present results are in agreement with previous studies that have investigated the effects of both SSGT and CAIT on soccer players and found beneficial effects in terms of vOBLA and Yo-Yo IR1 measures, exercise intensity during the intervention programs may discriminate this study from others. Our approach is not consistent with the suggestion made by several authors that training at intensities above $90 \%$ of the HRmax is preferable for enhancing endurance capacity (Ferrari Bravo, et al., 2008; Helgerud, et al., 2001; Hill-Haas, et al., 2009; Impellizzeri, et al., 2006; McMillan, et al., 2005; Reilly \& White, 2005). The extend of the changes in aerobic performance that follow a training program depends on various factors, exercise intensity of the intervention included (Helgerud, et al., 2007). However, in our study, HR or exercise intensity that was employed was HR ( \pm 5 beats $/ \mathrm{min}$ ) that corresponded to the lactate threshold level of $4 \mathrm{mmol} \cdot \mathrm{L}^{-1}$ (OBLAHR) of each individual. Similar improvements in the vOBLA and Yo-Yo IR1 performance in both training groups of the present study may be related to similar duration of exercise and individualized exercise intensities. Other factors that may influence magnitude of the response to training are the intervention duration, the baseline fitness levels of the individuals and the time of the season (Bogdanis, Ziagos, Anastasiadis, \& Maridaki, 2007; Buchheit, et al., 2009; Hill-Haas, et al., 2009; Impellizzeri, et al., 2006). The percent increases in the Yo-Yo IR1 performance levels were $89.1 \%$ in the CAITG and $63.1 \%$ in the SSGTG. The low baseline endurance values of the participants in this study may partly explain large percentage changes in the Yo-Yo IR1 performance. These factors should be taken into account when interpreting the results.

Soccer is characterized as a high-intensity intermittent team sport (Hoff, et al., 2002). The Yo-Yo IR tests provide more sensitive measures of changes in performance in intermittent sports than $\mathrm{VO}_{2 \max }$ tests (Bangsbo, et al., 2008). SSGs more closely resemble actual soccer game than interval running due to their intermittent structure. Although the SSGT intervention program was expected to lead to greater improvements in Yo-Yo IR1 performance than the CAIT intervention program, the improvements in the Yo-Yo IR1 performance (i.e., soccerspecific endurance) following SSGT and CAIT were similar. 
As expected, the SSGT significantly improved short-passing abilities, as assessed by the LSPT, while the CAIT did not (Table 3). When performing the LSPT, it is possible that the players benefited from the favorable effects of SSGT, which involved short passing. Here, short-passing ability was measured by the LSPT, a valid and reliable test (Ali, et al., 2007), but previous studies investigated the effects of SSGT on specific technical soccer skills using different test methods, such as ball control (i.e., juggling), passing, and dribbling skills, etc. Different test methods for measuring soccer-specific technical skills may elicit different training-induced improvement results that could cause discrepancies between this and other studies. A few authors have highlighted that the main benefits of SSGT and interval training are the improvement or maintenance of soccer-specific technical skills (Helgerud, et al., 2001; Radziminski, et al., 2013; Reilly \& White, 2005).

Other studies have produced similar results. After eight weeks of intervention training, Radziminski et al. (2013) observed a significant improvement in the soccer-specific technical skill level in the SSGT group $\left(4 \times 4\right.$ min over $90 \%$ of the $\mathrm{HR}_{\max }$ with $3 \mathrm{~min}$ of active recovery) and no improvement in the running group. Levels of soccer-specific technical skills, such as ball control (i.e., juggling), passing (i.e., against the bench, to the goal, rotation passes, long passes, heading), and dribbling were evaluated using the battery of tests proposed by the German Football Federation (Radziminski, et al., 2013). In the study done by Reilly and White (2005), the football dribbling ability was measured both before and after the interval and SSG training and no deterioration in dribbling ability was observed in either group. The discrepancy between the present study and that of Reilly and White (2005) is that the authors observed no deterioration in dribbling ability, whereas the present study observed improvements in short-passing ability. Previously, a few authors have investigated the influence of high-intensity interval training on the soccer technique level. Helgerud et al. (2001) examined changes in performance on a technical test after an 8-week program of interval training (i.e., $4 \times 4$ minutes of work at $90-95 \%$ of $\mathrm{HR}_{\max }$ with 3 minutes of active recovery twice per week) and observed no improvements in kicking precision or kicking velocity from 16 meters to the goal. In the present study, the SSGT group performed a total of 11282 offensive skills (ball control, passing and dribbling), while the CAIT group did not perform these skills during the time of intervention. Because the time spent in the games with the ball was greater for the SSGT group during the intervention, it is not surprising that the short-passing ability values during the LSPT were significantly higher in the SSGT group.
The interventions' effects on offensive and defensive skills were analyzed during matches. The results revealed greater benefits of SSGT than CAIT in terms of defensive and offensive skills execution during the observed matches. One possible reason for these noticeable improvements in both skills in the SSGTG may be that the players were accustomed to soccer-specific exercises. A significantly greater number of offensive skills exhibited by the SSGTG during the matches may be attributable to a greater amount of time devoted to the work with the ball during the SSG training than the CAI training. The lack of improvement in defensive skills in the CAITG can be explained by the fact that the CAIT involved no ball and no opponent to create pressure. While the absence of similar studies in the soccer literature prevents comparisons of our results to those of other studies, one basketball study highlighted greater benefits of SSG compared with high-intensity interval training in terms of matchspecific actions including defensive and offensive skills (Delextrat \& Martinez, 2014). SSGs have also been suggested to facilitate the development of technical skills and tactical awareness within contexts appropriate to the game (Allison \& Thorpe, 1997; Little \& Williams, 2006). An investigation in the basketball literature that is similar to our study revealed that small-sided games were sufficient to improve basketball-specific skills (i.e., shooting skills). However, in contrast to our findings, this basketball study found that passing skills were similarly increased by both high-intensity interval training and SSGT (Delextrat \& Martinez, 2014). In this study, one of the main differences between the two training methods was that the presence of the ball during the small-sided games allowed for concomitant improvements in technical and tactical skills and increased player motivation (Flanagan \& Merrick, 2002; Iaia, Rampinini, \& Bangsbo, 2009).

The pre- and post-test and match results of both groups showed changes; other than the intervention, the contents of the practices were the same for both groups. Since the only activity executed by the players other than the soccer practices was the intervention program, it is highly possible that the intervention may be the reason for the pre-post changes.

We conclude that various defensive and offensive actions (e.g., tackles, air duels, and passing) that occur during SSGT may be responsible for the observed significant improvement in defensive and offensive skills and passing performance during the test matches and passing tests.

The results of this study suggested that six weeks of SSGT improved short-passing ability, as assessed with the LSPT, defensive and offensive soccer skills during match, and various physiological parameters (i.e., endurance parameters), while CAIT only resulted in improvements 
in physiological parameters. SSGT concurrently improves soccer specific endurance and technicaltactical ability, thus it is highly time efficient. While engaging in small-group play, soccer players are more motivated by playing with the ball and no longer having to perform plain running to improve their soccer-specific endurance and offensive and defensive skills. The limitations of the study were the absence of the control group and participants' low baseline endurance values. Furthermore, more research on the respective subject with fitter players and the control group is necessary.

\section{References}

Ali, A. (2011). Measuring soccer skill performance: A review. Scandinavian Journal of Medicine and Science in Sports, 21(2), 170-183.

Ali, A., Williams, C., Hulse, M., Strudwick, A., Reddin, J., Howarth, L., Eldred, J., Hirst, M., \& McGregor, S. (2007). Reliability and validity of two tests of soccer skill. Journal of Sports Sciences, 25(13), 1461-1470.

Allison, S., \& Thorpe, R. (1997). A comparison of the effectiveness of two approaches to teaching games within physical education: A skills approach versus a games for understanding approach. British Journal of Physical Education, 28(3), 9-13.

Bangsbo, J., Iaia, F.M., \& Krustrup, P. (2008). The Yo-Yo Intermittent Recovery Test: A useful tool for evaluation of physical performance in intermittent sports. Sports Medicine, 38(1), 37-51.

Bekris, E., Gissis, I., Sambanis, M., Milonys, E., Sarakinos, A., \& Anagnostakos, K. (2012). The physiological and technical-tactical effects of an additional soccer player's participation in small sided games training. Electronic Journals of Martial Arts and Sciences - Physical Training. Retrieved January 15, 2014 from: http://ejmas.com/ $\mathrm{pt} / \mathrm{ptframe} . \mathrm{html}$

Bogdanis, G.C., Ziagos, V., Anastasiadis, M., \& Maridaki, M. (2007). Effects of two different short-term training programs on the physical and technical abilities of adolescent basketball players. Journal of Science and Medicine in Sport, 10(2), 79-88.

Bradley, P., O’Donoghue, P., Wooster, B., \& Tordoff, P. (2007). The reliability of ProZone MatchViewer: A video-based technical performance analysis system. International Journal of Performance Analysis in Sport, 7(3), 117-129.

Buchheit, M., Laursen, P.B., Kuhnle, J., Ruch, D., Renaud, C., \& Ahmaidi, S. (2009). Game-based training in young elite handball players. International Journal of Sports Medicine, 30(4), 251-258.

Chamari, K., Hachana, Y., Kaouech, F., Jeddi, R., Moussa-Chamari, I., \& Wisløff, U. (2005). Endurance training and testing with the ball in young elite soccer players. British Journal of Sports Medicine, 39(1), 24-28.

Cohen, J. (1988). Statistical power analysis for the behavioral sciences $\left(2^{\text {nd }}\right.$ ed.). Hillsdale, NJ: Lawrence Erlbaum Associates.

Coolican, H. (2009). Research methods and statistics in psychology (5 $5^{\text {th }}$ ed.). London: Hodder Education.

da Silva, C.D., Impellizzeri, F.M., Natali, A.J., De Lima, J.R.P., Bara, M.G., Silami-Garcia, E., \& Marins, J.C.B. (2011). Exercise intensity and technical demands of small-sided games in young Brazilian soccer players: Effect of number of players, maturation, and reliability. Journal of Strength and Conditioning Research, 25(10), 2746-2751.

Delextrat, A., \& Martinez, A. (2014). Small-sided game training improves aerobic capacity and technical skills in basketball players. International Journal of Sports Medicine, 35(5), 385-391.

Dellal, A., Chamari, K., Pintus, A., Girard, O., Cotte, T., \& Keller, D. (2008). Heart rate responses during small-sided games and short intermittent running training in elite soccer players: A comparative study. Journal of Strength and Conditioning Research, 22(5), 1449-1457.

Dellal, A., Owen, A., Wong, D.P., Krustrup, P., Van Exsel, M., \& Mallo, J. (2012). Technical and physical demands of small vs. large sided games in relation to playing position in elite soccer. Human Movement Science, 31(4), 957-969.

Eniseler, N. (2005). Heart rate and blood lactate concentrations as predictors of physiological load on elite soccer players during various soccer training activities. Journal of Strength and Conditioning Research, 19(4), 799-804.

Fanchini, M., Azzalin, A., Castagna, C., Schena, F., McCall, A., \& Impellizzeri, F.M. (2011). Effect of bout duration on exercise intensity and technical performance of small-sided games in soccer. Journal of Strength and Conditioning Research, 25(2), 453-458.

Ferrari Bravo, D., Impellizzeri, F.M., Rampinini, E., Castagna, C., Bishop, D., \& Wisloff, U. (2008). Sprint vs. interval training in football. International Journal of Sports Medicine, 29(8), 668-674.

Flanagan, T., \& Merrick, E. (2002). Holistic training: Quantifying the work-load of soccer players. In A. Murphy, T. Reilly \& W. Spinks (Eds.), Science and Football IV (pp. 341-349). London and New York: Routledge.

Guner, R., Kunduracioglu, B., \& Ulkar, B. (2006). Running velocities and heart rates at fixed blood lactate concentrations in young soccer players. Advances in Therapy, 23(3), 395-403.

Helgerud, J., Engen, L., Wisløff, U., \& Hoff, J. (2001). Aerobic endurance training improves soccer performance. Medicine and Science in Sports and Exercise, 33, 1925-1931. 
Helgerud, J., Hoydal, K., Wang, E., Karlsen, T., Berg, P., Bjerkaas, M., ... Hoff, J. (2007). Aerobic high-intensity intervals improve $\mathrm{VO}_{2 \max }$ more than moderate training. Medicine and Science in Sports and Exercise, 39(4), 665-671.

Hill-Haas, S.V., Coutts, A.J., Rowsell, G.J., \& Dawson, B.T. (2009). Generic versus small-sided game training in soccer. International Journal of Sports Medicine, 30(9), 636-642.

Hoff, J., Wisløff, U., Engen, L.C., Kemi, O.J., \& Helgerud, J. (2002). Soccer specific aerobic endurance training. British Journal of Sports Medicine, 36(3), 218-221.

Iaia, M.F., Rampinini, E., \& Bangsbo, J. (2009). High-intensity training in football. International Journal of Sports Physiology and Performance, 4(3), 291-306.

Ian, J. (2004). The use of small-sided games in the metabolic training of high school soccer players. Strength and Conditioning Journal, 26(5), 11-18.

Impellizzeri, F.M., Marcora, S.M., Castagna, C., Reilly, T., Sassi, A., Iaia, F.M., \& Rampinini, E. (2006). Physiological and performance effects of generic versus specific aerobic training in soccer players. International Journal of Sports Medicine, 27(6), 483-492.

Krustrup, P., Mohr, M., Amstrup, T., Rysgaard, T., Johansen, J., Steensberg, A., Pedersen P.K., \& Bangsbo, J. (2003). The Yo-Yo intermittent recovery test: Physiological response, reliability, and validity. Medicine and Science in Sports and Exercise, 35(4), 697-705.

Little, T., \& Williams, A.G. (2006). Suitability of soccer training drills for endurance training. Journal of Strength and Conditioning Research, 20(2), 316-319.

Liu, H., Gomez, M.Á., Lago-Peñas, C., \& Sampaio, J. (2015). Match statistics related to winning in the group stage of 2014 Brazil FIFA World Cup. Journal of Sports Sciences, 33(5), 1205-1213.

Liu, H., Hopkins, W., Gomez, M. A., \& Molinuevo, J. S. (2013). Inter-operator reliability of live football match statistics from OPTA Sportsdata. International Journal of Performance Analysis in Sport, 13(3), 803-821.

Martone, D., Giacobbe, M., Capobianco, A., Imperlini, E., Mancini, A., Capasso, M., Buono, P., \& Orrù, S. (2017). Exercise intensity and technical demands of small-sided soccer games for under-12 and under-14 players: Effect of area per player. Journal of Strength and Conditioning Research, 31(6), 1486-1492.

McMillan, K., Helgerud, J., Macdonald, R., \& Hoff, J. (2005). Physiological adaptations to soccer specific endurance training in professional youth soccer players. British Journal of Sports Medicine, 39(5), 273-277.

O’Donoghue, P. (2007). Reliability issues in performance analysis. International Journal of Performance Analysis, $7(1), 35-48$.

OPTA. (2013). Definitions OPTA and pack training 2012/13. Retrieved February 20, 2014 from http://optasports.com/ news-area/blog-optas-event-definitions.aspx

Radziminski, L., Rompa, P., Barnat, W., Dargiewicz, R., \& Jastrzebski, Z. (2013). A comparison of the physiological and technical effects of high-intensity running and small-sided games in young soccer players. International Journal of Sports Science \& Coaching, 8(3), 455-465.

Rampinini, E., Impellizzeri, F.M., Castagna, C., Abt, G., Chamari, K., Sassi, A., \& Marcora, S.M. (2007). Factors influencing physiological responses to small-sided soccer games. Journal of Sports Sciences, 25(6), 659-666.

Reilly, T. (1996). Motion analysis and physiological demands. In T. Reilly \& A.M. Willams (Eds.), Science and soccer (pp. 65-79). London: E \& FN Spon.

Reilly, T., \& White, C. (2005). Small-sided games as an alternative to interval-training for soccer players. In T. Reilly, J. Cabri \& D. Araújo (Eds.), Science and Football V (pp. 344-347). London and New York: Routledge.

Sassi, R., Reilly, T., \& Impellizzeri, F. (2005). Comparison of small-sided games and interval training in elite professional soccer players. In T. Reilly, J. Cabri \& D. Araújo (Eds.), Science and Football V (pp. 341-243). London: E \& FN Spon.

Wisløff, U., Helgerud, J., \& Hoff, J. (1998). Strength and endurance of elite soccer players. Medicine and Science in Sports and Exercise, 30(3), 462-467.

Yuhasz, M. (1962). The effects of sports training on body fat in man with predictions of optimal body weight. Urbana: University of Illinois.

Submitted: April 5, 2016

Accepted: August 3, 2017

Published Online First: November 10, 2017

Correspondence to:

Niyazi Eniseler, Ph.D.

Faculty of Sports Sciences,

Celal Bayar University, Manisa, Turkey

Phone: + 905334679975

Fax: + 902362313001

E-mail: niyazieniseler66@gmail.com 